\title{
Radii of Rydberg states of isolated silicon donors
}

\author{
Juerong Li, ${ }^{1}$ Nguyen H. Le, ${ }^{1}$ K. L. Litvinenko, ${ }^{1}$ S. K. Clowes, ${ }^{1}$ H. Engelkamp, ${ }^{2}$ S. G. Pavlov, ${ }^{3}$ H.-W. Hübers, ${ }^{3,4}$ \\ V. B. Shuman, ${ }^{5}$ L. M. Portsel,${ }^{5}$ A. N. Lodygin, ${ }^{5}$ Yu. A. Astrov, ${ }^{5}$ N. V. Abrosimov ${ }^{6}$ C. R. Pidgeon, ${ }^{7}$ A. Fisher, ${ }^{8}$ Zaiping Zeng, ${ }^{9}$ \\ Y.-M. Niquet, ${ }^{9}$ and B. N. Murdin ${ }^{1, *}$ \\ ${ }^{1}$ Advanced Technology Institute, University of Surrey, Guildford, GU2 7XH, United Kingdom \\ ${ }^{2}$ High Field Magnet Laboratory (HFML-EMFL), Radboud University, Toernooiveld 7, 6525 ED Nijmegen, The Netherlands \\ ${ }^{3}$ Institute of Optical Sensor Systems, German Aerospace Center (DLR), Rutherfordstrasse 2, 12489 Berlin, Germany \\ ${ }^{4}$ Humboldt Universität zu Berlin, Institut für Physik, Newtonstrasse 15, 12489 Berlin, Germany \\ ${ }^{5}$ Ioffe Institute, Russian Academy of Sciences, St. Petersburg, Russia \\ ${ }^{6}$ Leibniz Institute for Crystal Growth, Max-Born-Straße 2, 12489 Berlin, Germany \\ ${ }^{7}$ Institute of Physics and Quantum Science, SUPA, Heriot-Watt University, Edinburgh, EH14 4AS, United Kingdom \\ ${ }^{8}$ London Centre for Nanotechnology and Department of Physics and Astronomy, University College London, \\ London WC1H OAH, United Kingdom \\ ${ }^{9}$ Université Grenoble Alpes, CEA, INAC-MEM, L_Sim 38000 Grenoble, France
}

(Received 21 March 2018; published 15 August 2018)

\begin{abstract}
We have performed high field magnetoabsorption spectroscopy on silicon doped with a variety of single and double donor species. The magnetic field provides access to an experimental magnetic length, and the quadratic Zeeman effect, in particular, may be used to extract the wave-function radius without reliance on previously determined effective mass parameters. We were, therefore, able to determine the limits of validity for the standard one-band anisotropic effective mass model. We also provide improved parameters and use them for an independent check on the accuracy of effective mass theory. Finally, we show that the optically accessible excited-state wave functions have the attractive property that interactions with neighbors are far more forgiving of position errors than (say) the ground state.
\end{abstract}

DOI: 10.1103/PhysRevB.98.085423

\section{INTRODUCTION}

Impurities in silicon provide a platform for classical microelectronics and quantum technology. Knowledge of the wave-function extent is needed for prediction of the interaction between donors and their neighbors for tests of physics [1-4], device transport [5] and entanglement/gating [6-9]. With knowledge of the extent, the atoms may be appropriately placed to optimize these interactions [10,11]. Qubit schemes being currently investigated that use excited states include a variety of species $[6,12]$ including double donors such as selenium [13]. Amazingly, in spite of their ubiquity and enormous technical importance, measurement of the state radius of any isolated silicon impurity is lacking after more than six decades of research [14]. Regular arrays are desired for quantum computer architectures [7-9], for which information on the neighbor-neighbor interactions will be crucial, just as it is for free atoms [15]. Because wave functions decay exponentially, a rapid change in the coupling occurs as a function donor-donor separation [4]; this is the single impurity equivalent of the Mott metal-insulator transition, where control of the coupling requires good information on the separation at which the change occurs. Indeed the simplest experimental way to access the wave-function extent is via the metal-insulator transition for ground states and a similar transition occurs for excited

${ }^{*}$ Corresponding author: b.murdin@surrey.ac.uk states [16], however, this is a complicated many-body problem and its precise details are unclear, so that it can only be used approximately for the ground state of single donors, and not at all for double donors (because they produce half-full and full impurity bands, respectively). Without knowledge of the wave-function extent we cannot engineer the contact of the impurity with readout electronics [5], external leads (source, drain, gates etc.) or know how much control is required to construct a dimer [4], a chain [6] or a lattice [3]. The questions we raise here are closely analogous to those for cold Rydberg atoms in magnetic traps, where the excited states are large and highly susceptible to magnetic fields (as in our case), and so are the dipole moments and interactions with neighboring atoms that affect both the spectra [17] and the formation of condensates [18], though in this case the ion is fixed, and we have the extra complication of an anisotropic effective mass.

Here, we show that the wave-function radius for excited states can be found directly from the ratio of the coefficients of the linear and quadratic Zeeman effects (LZEs and QZEs) without the need for any effective mass parameters, and provide a radius measurement of hydrogenic impurity excited states. Effective mass theory (EMT) [14,19-21,23] may be used to predict the spectrum and the wave-function radius from three parameters; two effective mass values and the permittivity. We provide a self-consistent set of parameters obtained only from the zero-field spectrum and LZE, and use the resulting prediction for the QZE as an independent check on the validity of EMT. 
There are currently two primary methods to detect wavefunction properties experimentally: via electron spin resonance (ESR), which measures contact with the donor nucleus, or via tunneling methods, which measure contact with the surface/barrier nearby. ESR [24-26] is excellent for determining the central part of the wave function but not necessarily the long-range part that would be responsible for coupling to neighbors. Recently, images of the ground-state wave function of near-surface impurities have been obtained from scanning tunneling microscopy (STM) [27-30], which allows direct observation of the density where the donor wave functions touch the surface. The images are difficult to interpret with high accuracy because the signal caused by the donor is a small modulation on top of the density due to the surface atoms, and a very careful Fourier transform filtering and other processing is required [28]. The tunneling spectroscopy of donors in contact with a barrier is also possible [31,32]. In either case, imaging and tunneling spectroscopy are limited to near-interface states that are naturally strongly perturbed. ESR and tunneling spectroscopy have only been used to extract the state radius for the ground state. Extraction of ground-state dimensions from the QZE is also possible, but more assumptions are required [33,34].

\section{EXPERIMENT}

In this paper, we investigate the excited-state wave-function extent from the magnetic length. We investigated the QZE in bulk-doped silicon with single substitutional donors $\mathrm{Bi}, \mathrm{Sb}$ or $\mathrm{P}[35,36]$, the single interstitial donor $\mathrm{Li}$, substitutional double donors $\mathrm{S}[37,38]$ and Se [33,39], the interstitial double donor $\mathrm{Mg}$ [40], and double donor complexes $\mathrm{S}_{2}$ and $\mathrm{Se}_{2}$. The doping of each was in the range $1 \times 10^{14}-2 \times 10^{15} \mathrm{~cm}^{-3}$, low enough that the distance between the donors is far larger than the orbit radius of any of the states of interest. We performed infrared transmission spectroscopy at $T=1.4 \mathrm{~K}$ as a function of magnetic field up to $B=30 \mathrm{~T}$, in the Faraday configuration. All the samples were cut into [001] wafers and beveled to $1^{\circ}$ to avoid Fabry-Pérot interference, and the resolution was $0.04 \mathrm{meV}$ determined by residual water vapor absorption lines. Data for the Si:P and Si:Li samples were resolution limited. The transmitted intensity was recorded as a function of frequency and field $I(v, B)$. The median of $I(v, B)$ across all magnetic fields at each frequency was used to find the field-independent background spectrum $I_{\text {background }}(v)$, and hence the transmission $T(v, B)=I(v, B) / I_{\text {background }}(v)$, as in the example of Fig. 1(a). See Ref. [35] for more experimental details. The transmission spectrum shows well-resolved absorption lines and clear evidence of the LZE (e.g., in the splitting of the $2 p_{+}$ and $2 p_{-}$transitions at low field) and the QZE (e.g., in the curvature of the $2 p_{-}$at high field).

\section{A. Perturbation theory for excited states}

Effective mass theory (EMT) [14,19-23] predicts hydrogenic donor states very well using length, energy, and field parameters $a_{B}^{*}=a_{B} \epsilon_{r} / m_{t}^{*}, E_{H}^{*}=E_{H} m_{t}^{*} / \epsilon_{r}^{2}$ and $B_{a}^{*}=$ $\hbar / e a_{B}^{* 2}=\hbar m_{t}^{* 2} / e a_{B}^{2} \epsilon_{r}^{2}$ that are scaled from the atomic hydrogen Bohr radius, Hartree energy, and atomic unit of magnetic field, respectively, by the relative effective mass $m_{t}^{*}$ and relative dielectric constant $\epsilon_{r}$. Silicon is indirect and the conduction
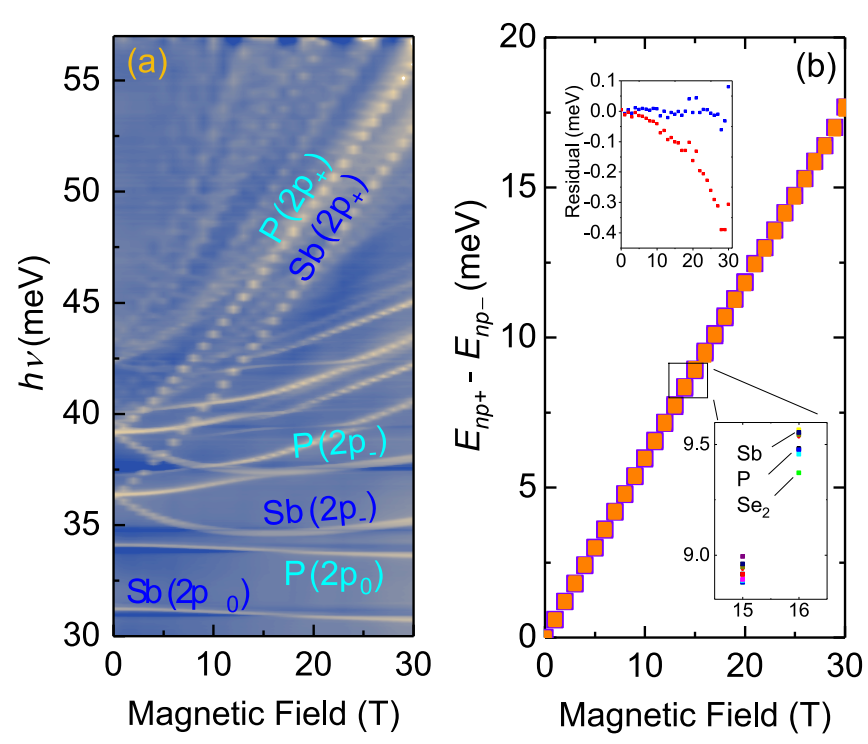

FIG. 1. Linear Zeeman effect. (a) Lyman series of the Si:P,Sb co-doped sample. Labels indicate the excited state for three of the strongest Lyman series transitions. The color scale indicates the transmission (dark blue $=$ high transmission; light yellow $=$ high absorption). (b) Splitting between the transitions for the same $n$ but a different $m$, i.e., $h v_{1 s \rightarrow n p_{+}}-h v_{1 s \rightarrow n p_{-}}=E_{n p_{+}}-E_{n p_{-}}$against $B$. Data presented are for $n=2$ and 3 for all species used in this paper ( $\mathrm{Si}: X$ where $X=\mathrm{Li}, \mathrm{P}, \mathrm{Sb}, \mathrm{Bi}, \mathrm{Mg}, \mathrm{Se}, \mathrm{Se}_{2}, \mathrm{~S}$ ) showing they all follow the same field dependence. Inset bottom: Expanded scale section from main panel showing 16 points at each field with a different color symbol for each species $/ n$ combination (and only three examples are labeled due to the small scatter). Inset top: Residuals from the linear fit (red) and from the nonparabolic model fit (blue) to the $\mathrm{Si}: \mathrm{P}$ data from the main panel.

band minimum is far from $k=0$ near the six equivalent $X$ points of the Brillouin zone (along the $\langle 001\rangle$ directions). These six valleys are anisotropic, characterized by a mass transverse to the valley axis $m_{t}^{*}$ and a mass anisotropy parameter $\gamma$ ( $=m_{t}^{*} / m_{l}^{*}$ where $m_{l}^{*}$ is the mass along the axis). According to the Kohn-Luttinger [19] EMT model, intervalley interactions are to be ignored (or treated later by perturbation theory), and the single-valley wave function is taken to be the product of a slowly varying envelope and quickly varying terms: $\psi_{j, \mu}(\mathbf{r})=$ $f_{j, \mu}(\mathbf{r}) e^{i \mathbf{k}_{\mu} \cdot \mathbf{r}}$ where $\mathbf{k}_{\mu}$ is the momentum at the bottom of the valley with index $\mu$ (i.e., $\mathbf{k}_{\mu}$ is along $x,-x, y,-y, z,-z$ and $\left|\mathbf{k}_{\mu}\right|=0.85 \pi / a=k_{0}$, where $a$ is the lattice constant) and $j$ is an index (or set of indices) identifying the state within the valley. Using single-valley EMT works well for the excitedstate energies [14] (and our aim here is to assess the accuracy of the radius prediction for the excited states). We ignored an additional lattice-periodic factor $[19,22,23]$ since it does not influence any of what follows or the intended application of engineering donor-donor interactions. The envelope functions $f_{j, \mu}$ are solutions of $\hat{H}_{\mu} f_{j, \mu}=\varepsilon_{j} f_{j, \mu}$. In the case that a magnetic field is applied parallel to the axis of the $z$ valley, the Hamiltonian for that valley is $[14,33-36]$

$$
\begin{aligned}
\hat{H}_{z}= & -\frac{E_{H}^{*} a_{B}^{* 2}}{2}\left[\frac{\partial^{2}}{\partial x^{2}}+\frac{\partial^{2}}{\partial y^{2}}+\gamma \frac{\partial^{2}}{\partial z^{2}}\right]-\frac{E_{H}^{*} a_{B}^{*}}{r}+\frac{E_{H}^{*} B L_{z}}{2 B_{a}^{*} \hbar} \\
& +\frac{E_{H}^{*} B^{2}\left(x^{2}+y^{2}\right)}{8 B_{a}^{* 2} a_{B}^{* 2}} .
\end{aligned}
$$


The first two terms comprise the zero-field Hamiltonian for a hydrogenic atom including the mass anisotropy $\hat{H}_{0 z}$. We neglect tetrahedral corrections to the impurity potential [41]. The last two are, respectively, the LZE and QZE terms $\hat{H}_{1}$ and $\hat{H}_{2}$. For other valleys and field directions the Hamiltonian is more complex and we shall not concern ourselves with such cases. Comparison of the eigenvalues of Eq. (1) with the experimental zero-field energy spectrum allows extraction/verification of $E_{H}^{*}$ and $\gamma$ only, and the LZE allows extraction of $B_{a}^{*}$. If it is assumed Eq. (1) holds, and therefore $B_{a}^{*}=\hbar / e a_{B}^{* 2}$, this is enough to predict $a_{B}^{*}$. In this paper we measure the ratio of the QZE and LZE, which is a means to extract the radius directly, and provides in essence experimental measurement of $B_{a}^{*} a_{B}^{* 2}$ as a test of the validity of Eq. (1). In other words, whereas the zero-field spectrum and LZE can provide tests of the scaling rules given above for $E_{H}^{*}$ and $B_{a}^{*}$, only the QZE can test the scaling rule for $a_{B}^{*}$ independently.

Equation (1) has cylindrical symmetry about $z$ so the azimuthal dependence of the wave-function envelope is $e^{i m \phi}$, which is an eigenfunction of the LZE term with quantum number $m$, the magnetic quantum number. For our field direction $(\mathbf{B} \| z) \hat{H}_{1}$ commutes with $\hat{H}_{0 z}$ and $\hat{H}_{2}$ so there are no off-diagonal matrix elements of $\hat{H}_{1}$, and the magnetic quantum number $m$ is conserved for all $B$. The LZE energy $E_{1}=\mu_{B}^{*} m B$ is, therefore, well defined for all $B . \hat{H}_{0 z}$ and $\hat{H}_{2}$ do not commute, but for sufficiently small field we can treat $\hat{H}_{2}$ by perturbation theory which produces

$$
E(B)=E_{0}+\mu_{B}^{*} m B+\frac{e \mu_{B}^{*}}{4 \hbar} \rho_{0}^{2} B^{2},
$$

where $\rho_{0}$ is the value of the transverse radius $\rho=\sqrt{\left\langle x^{2}+y^{2}\right\rangle}$ at zero field. The effective Bohr magneton $\mu_{B}^{*}=E_{H}^{*} / 2 B_{a}^{*}=$ $e E_{H}^{*} a_{B}^{* 2} / 2 \hbar=\mu_{B} / m_{t}^{*}$, and we substituted $E_{H}^{*} / B_{a}^{* 2} a_{B}^{* 2}=$ $2 e \mu_{B}^{*} / \hbar$. As we shall see, $E(B)$ becomes nonparabolic at high field because $\rho$ shrinks due to magnetic confinement so that the small perturbation approximation fails (when the last term in Eq. (2) becomes significant compared with the first), and also because of a very slight field dependence of $m_{t}^{*}$. In this case $\hat{H}_{0 z}$ and $\hat{H}_{2}$ are mixed and their contributions cannot be separated. We define an effective transverse radius, $\tilde{\rho}$, given by

$$
\frac{d^{2} E}{d B^{2}}=\frac{e \mu_{B}^{*}}{2 \hbar} \tilde{\rho}^{2},
$$

which is equal to the actual transverse radius at low field, i.e., $\tilde{\rho}^{2}(0)=\rho_{0}^{2}$ as shown by Eq. (2). It is useful to note that $\hat{H}_{0 z}$ and $\hat{H}_{2}$ have the same symmetry for $\mathbf{B} \| z$, so solving eigenvalues and eigenfunctions of Eq. (1) for $B \neq 0$ is no more difficult than for $B=0$. For other field directions $\hat{H}_{0 z}, \hat{H}_{1}$, and $\hat{H}_{2}$ are all mutually noncommuting and $m$ is not a good quantum number.

The ratio of the coefficients of the QZE and LZE in Eq. (2) contains $\rho_{0}^{2}$ and fundamental constants but not the effective masses etc., which suggests a convenient way to determine the radius.

\section{B. Linear Zeeman effect}

We require the ratio of the QZE and LZE terms in Eq. (2), and we start with the LZE. It is easy to extract $\mu_{B}^{*}$ directly from the experimental field dependence for $\mathbf{B} \| z$ because the
TABLE I. Effective mass parameters obtained.

\begin{tabular}{lc}
\hline \hline Fit parameters & Value \\
\hline$\mu_{B}^{*}$ & $0.2978 \pm 0.0003 \mathrm{meV} / \mathrm{T}$ \\
$B_{n p}$ & $242 \pm 12 \mathrm{~T}$ \\
$\gamma$ & $0.2096 \pm 0.0002$ \\
$E_{H}^{*}$ & $39.83 \pm 0.03 \mathrm{meV}$ \\
\hline Inferred parameters & Value \\
$m_{t}^{*}$ & $0.1944 \pm 0.0002$ \\
$m_{l}^{*}$ & $0.927 \pm 0.001$ \\
$\epsilon_{r}$ & $11.52 \pm 0.01$ \\
$a_{B}^{*}$ & $3.137 \pm 0.004 \mathrm{~nm}$ \\
$B_{a}^{*}$ & $66.88 \pm 0.09 \mathrm{~T}$ \\
\hline \hline
\end{tabular}

linear Zeeman energy is well defined and $m$ is a good quantum number: we simply take the difference between the transition energies to the $n p_{+}$and $n p_{-}$excited states, $h v_{1 s \rightarrow n p_{+}}$ $h v_{1 s \rightarrow n p_{-}}=E_{n p_{+}}-E_{n p_{-}}=2 \mu_{B}^{*}$. Since $\hat{H}_{1}$ commutes with $\hat{H}_{0 z}$ and $\hat{H}_{2}$, the quadratic and zero-field terms cancel exactly. It can be seen from Fig. 1(b) that this linear relationship holds very well, and the slope $2 \mu_{B}^{*}=2 \mu_{B} / m_{t}^{*}$ holds for all species and for both $2 p_{ \pm}$and $3 p_{ \pm}$excited states. At the highest fields used, there is a slight departure from linearity - the slope decreases at higher energy [Fig. 1(b) inset shows the residuals] that must have resulted from an effective mass increase. We presume that the mass increase arises due to the higher-frequency Fourier components in the envelope function introduced by the constriction with field, and therefore the increase is equal for both $p_{+}$and $p_{-}$states with the same $n$. If the mass rises with field and is an even function, a suitable nonparabolicity correction to the form of Fig. 1(b) is $E_{n p_{+}}-E_{n p_{-}}=2 \mu_{B}^{*} B /\left(1+B^{2} / B_{n p}^{2}\right)$ and a fit to the $\mathrm{Si}$ : P $2 p_{ \pm}$data gives the values of $\mu_{B}^{*}$ and $B_{n p}$ in Table I, which also shows the inferred value of $m_{t}^{*}$.

Our value of $m_{t}^{*}$ is $2.0 \pm 0.1 \%$ larger than the band-edge value, $0.1905 \pm 0.0001$ from cyclotron resonance for free electrons [20] (and closer to that derived from the approach of Fig. 1(b) by others, $0.195 \pm 0.002$ [42], though with higher precision here); this is also presumably due to the nonparabolicity, since the appropriate value for a donor is an average over a region of $k$ space around the conduction band minimum, the extent of which is given by the reciprocal of the wave function radius and evidently includes enough $k$ space to noticeably increase $m_{t}^{*}$. Applying the same fit procedure to the $\mathrm{Si}: \mathrm{P} 3 p_{ \pm}$ data produces a value of $\mu_{B}^{*}$ that is $1.3 \pm 0.1 \%$ larger than the band edge value, i.e., the difference is less than for $2 p_{ \pm}$as would be expected for a state that is larger in real space and smaller in reciprocal space.

\section{Quadratic Zeeman effect}

It is also easy to extract the transverse radius directly from the experiment. The second derivative of the transition energy is, from Eq. (3), $\frac{2 \hbar}{e \mu_{B}^{*}} \frac{d^{2}}{d B^{2}} h v_{n p_{ \pm}}=\frac{2 \hbar}{e \mu_{B}^{*}} \frac{d^{2}}{d B^{2}}\left[E_{n p_{ \pm}}-E_{1 s}\right]=$ $\tilde{\rho}_{n p_{ \pm}}^{2}-\tilde{\rho}_{1 s}^{2}$. Since $\hat{H}_{1}$ commutes with $\hat{H}_{0 z}$ and $\hat{H}_{2}$, the radius and its constriction with field do not depend on $m$, and hence the transverse radius at zero field $\rho_{n p_{-}}^{2}(0)=\rho_{n p_{+}}^{2}(0)$. Therefore, in order to extract the double derivative at $B=0$ more accurately 

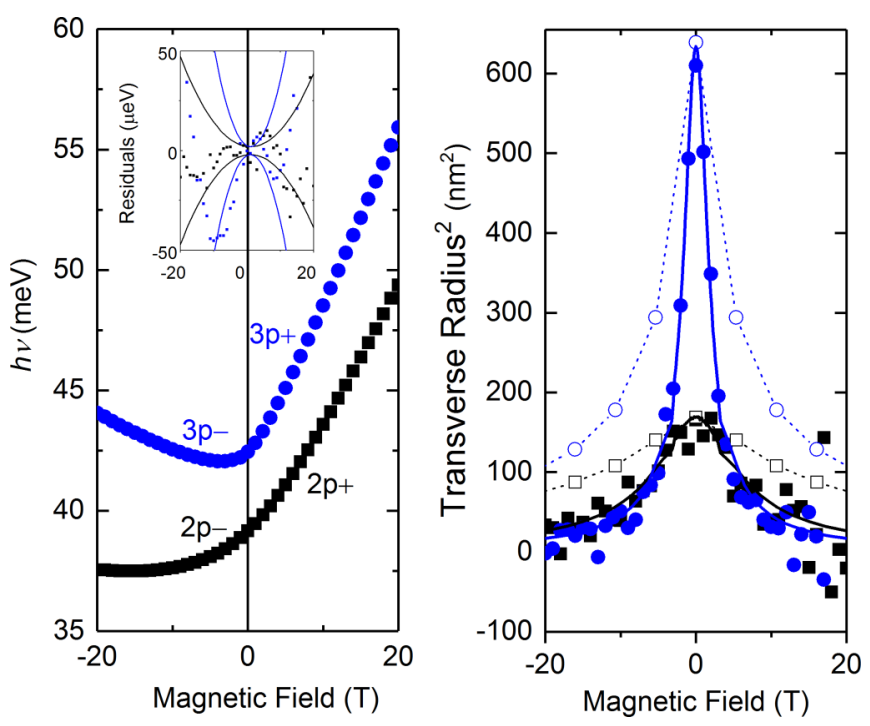

FIG. 2. Quadratic Zeeman effect. (a) Transition energy, $h v_{1 s \rightarrow n p_{ \pm}}$, for $\mathrm{Si}: \mathrm{P}$ for $2 p_{ \pm}$(squares) and $3 p_{ \pm}$(circles), with $m=+1$ transitions shown at positive field and $m=-1$ transitions shown at negative field. The fits described in the text produced the residuals shown as an inset, along with the weighting function used. (b) The transverse radius. The effective transverse radius squared for the transition, i.e., $\tilde{\rho}_{n p_{ \pm}}^{2}-\tilde{\rho}_{1 s}^{2}$ found by applying Eq. (3) to the experimental $h v_{1 s \rightarrow n p_{ \pm}}(B)$ shown in (a), (solid symbols) using the Savitzky-Golay method. Also shown is the theoretical effective transverse radius, i.e., Eq. (3) applied to $E_{n p_{ \pm}}(B)$ from EMT with the parameters from Table I (not a fit), solid lines. The open symbols show $\rho^{2}$ the actual (as opposed to effective) transverse radius squared $\left\langle x^{2}+y^{2}\right\rangle$ of the excited states from the EMT wave functions (with the same parameters, not a fit). The effective radius $\tilde{\rho}$ and actual radius $\rho$ are clearly the same at small field.

we may plot the transition energy vs field for $1 s \rightarrow n p_{+}$and $1 s \rightarrow n p_{-}$back to back [Fig. 2(a)]. The experimental results for $\tilde{\rho}_{n p_{ \pm}}^{2}-\tilde{\rho}_{1 s}^{2}$ for Si:P is shown in Fig. 2(b). Although we can see approximately the zero-field value from the figure, data extracted from derivatives of experimental data are always noisy, and it is preferable to extract the radius from fitting the raw data. We therefore need an analytical approximation for the QZE. Noting that the experimental dependence on Fig. 2(b) resembles a Lorentzian with zero-field value $\rho_{0}^{2}$, i.e., $\tilde{\rho}^{2} \approx \rho_{0}^{2}\left(1+B^{2} / B_{c}^{2}\right)^{-1}$, where $B_{c}$ is a parameter describing the field scale at which the constriction occurs, a suitable form is $E_{2}(B)=\frac{e \mu_{B}^{*}}{2 \hbar} \rho_{0}^{2} B_{c}^{2} g\left(B / B_{c}\right)$ where $g(x)=x \arctan (x)-$ $\frac{1}{2} \ln \left(x^{2}+1\right)$ (the double derivative of which produces the desired Lorentzian). We therefore performed a fit of

$$
E(B)=E_{0}+\frac{\mu_{B}^{*}}{1+B^{2} / B_{n p}^{2}}\left[B+\frac{e}{2 \hbar} \rho_{0}^{2} B_{c}^{2} g\left(\frac{B}{B_{c}}\right)\right]
$$

with free parameters $\rho_{0}, E_{0}$, and $B_{c}$ (and fixed $B_{n p}, \mu_{B}^{*}$ determined above). Crucially the factor $e \rho_{0}^{2} / 2 \hbar$, i.e., the ratio of the coefficients of the linear term and the QZE, does not depend on any effective mass parameters. The fit was weighted towards the data around $B=0$ (since this is where $\rho^{2}=\tilde{\rho}^{2}$ ) with a quadratic weighting function shown in the inset of Fig. 2(a) along with the residuals. We obtained values for the zero-field radius of $\rho_{2 p_{ \pm}}^{2}-\rho_{1 s}^{2}=159 \pm 1 \mathrm{~nm}^{2}$ and
TABLE II. Tight Binding results. The zero-field 3D radius $r_{1 s(A 1)}=\sqrt{3\left\langle z^{2}\right\rangle}$ (and the $2 \mathrm{D}$ radius $\rho_{1 s(A 1)}=\sqrt{\frac{2}{3}} r_{1 s(A 1)}$ ) were calculated from the TB wavefunctions. The QZE field tuning constant was found by calculating the binding energy from 0 to $30 \mathrm{~T}$ and fitting with a quadratic. For the conversion to $\tilde{\rho}_{A 1}^{2}$, we used the value of $\mu_{B}^{*}$ in Table I.

\begin{tabular}{lcccc}
\hline \hline & $r_{1 s(A 1)}(\mathrm{nm})$ & $\rho_{1 s(A 1)}(\mathrm{nm})$ & $\frac{e \mu_{B}^{*}}{4 \hbar} \tilde{\rho}_{1 s(A 1)}^{2}\left(\mathrm{neV} / \mathrm{T}^{2}\right)$ & $\tilde{\rho}_{1 s(A 1)}^{2}\left(\mathrm{~nm}^{2}\right)$ \\
\hline $\mathrm{P}$ & 2.481 & 2.026 & 255 & 2.240 \\
$\mathrm{As}$ & 2.125 & 1.735 & 181 & 1.590 \\
$\mathrm{Sb}$ & 2.608 & 2.130 & 284 & 2.495 \\
$\mathrm{Bi}$ & 1.630 & 1.331 & 99 & 0.870 \\
\hline \hline
\end{tabular}

$\rho_{3 p_{ \pm}}^{2}-\rho_{1 s}^{2}=611 \pm 5 \mathrm{~nm}^{2}$, and the corresponding values of $E_{0}$, the zero-field transition energy, were $39.161 \pm 0.001 \mathrm{meV}$ and $42.453 \pm 0.001 \mathrm{meV}$, respectively.

\section{Separating the ground-state contribution to the transition QZE}

For hydrogen we expect $\rho_{1 s}^{2} / \rho_{n p_{ \pm}}^{2}=n^{-4}$ and in Si:P the $1 s$ radius is further reduced by the central-cell correction (CCC) - a short-range potential that includes changes to the Coulomb potential where the electron penetrates into the ion core and increases the binding energy, so $\rho_{1 s}^{2}$ contributes negligibly to the QZE of the $1 s \rightarrow n p_{ \pm}$transition. In this approximation $\rho_{2 p_{ \pm}}=12.61 \pm 0.03 \mathrm{~nm}$ and $\rho_{3 p_{ \pm}}=24.7 \pm 0.1 \mathrm{~nm}$. The ratio of these values is not exactly $4 / 9$ simply because of the effect of mass anisotropy. Note that so far we have not used any EMT calculations or any assumed effective mass parameters, we only took the form of Eq. (2) to be correct.

The effective mass approximation is not expected to hold for the ground state, which is small, and is also subject to the CCC. The precise functional form of the CCC is unknown; only its symmetry and the end effect on the energy of the ground state are known. EMT is therefore untrustworthy for the ground state. The CCC mixes the six valley $1 s$ states, and the resulting lowest-energy component is the one labeled $1 s\left(A_{1}\right)$ (except in the case of $\mathrm{Si}: \mathrm{Li}$ ). Because this mixing introduces valleys transverse to the field for which Eqs. 1 and 2 do not hold, $\tilde{\rho}_{1 s(A 1)}(0)$ is not simply related to the actual zerofield transverse radius $\rho_{1 s(A 1)}$. We performed tight-binding calculations [43] in the range 0-30 T, and extracted values of the radius, and of the effective transverse radius by fitting a quadratic to $E_{1 s(A 1)}(B)$, as shown in Table II. The calculations were done with the $s p^{3} d^{5} s^{*}$ model [44], in supercells with side $L=48 a=26 \mathrm{~nm}$. On-site corrections were included on the impurity atom [45]. We can see that $\tilde{\rho}_{1 s(A 1)}^{2}(0)$ is about $1 \%$ of $\rho_{n p_{ \pm}}^{2}-\tilde{\rho}_{1 s(A 1)}^{2}(0)$ or less (note we abbreviated $\tilde{\rho}_{1 s(A 1)}^{2}$ to $\rho_{1 s}^{2}$ at the end of the previous section), and this confirms that it may be neglected for the purpose of studying the excited states.

\section{E. Exact diagonalization of single valley QZE}

We also investigated the detailed predictions of EMT by finding the eigenvalues and eigenfunctions of Eq. (1). The three independent parameters that are required in Eq. (1) are $E_{H}^{*}$, $a_{B}^{*}$, and $\gamma$ (recall that $B_{a}^{*}=\hbar / e a_{B}^{* 2}$ ), which may be found 


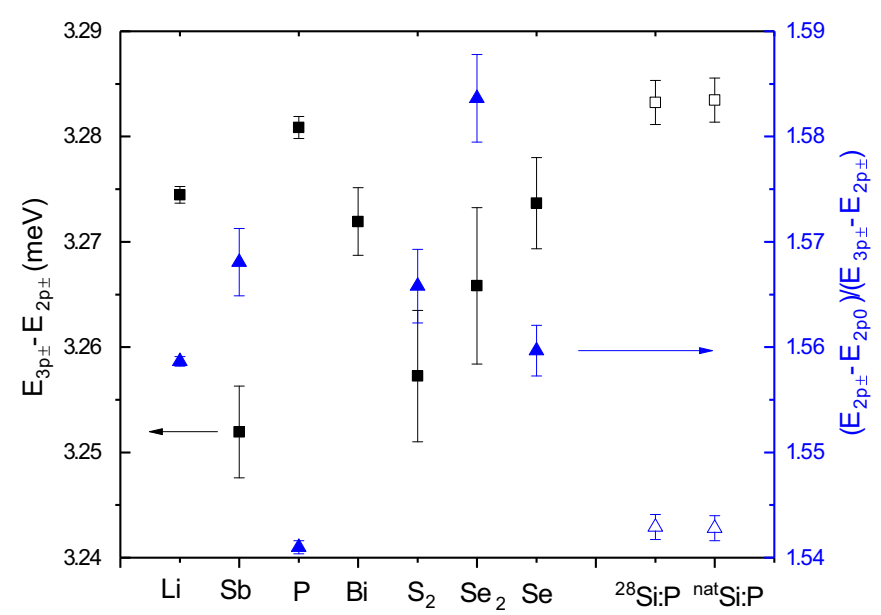

FIG. 3. Experimentally determined values of the zero-field energy splittings between excited states for different donor centers in silicon. Centers are displayed in order of binding energy. Data from Ref. [46] are also included as open symbols. Error bars are from Gaussian fits (this paper, or in the case of Ref. [46] the instrumental resolution).

from $\epsilon_{r}, m_{t}^{*}$, and $\gamma$ or vice versa. We follow the procedure of Faulkner [21] to extract $E_{H}^{*}$ (which determines the energy scale) and $\gamma$ (which determines the fractional splitting between the $p_{0}$ and $p_{ \pm}$states) by comparison with the zero-field energy spectrum with the eigenvalues of $\hat{H}_{0 z} f_{j, z}=\varepsilon_{j} f_{j, z}$ (i.e., in zero field), except that whereas Faulkner used a multivariate minimization of variational solutions, we calculated $f$ with a Lanczos method [35] (although our theoretical results for $E_{n p m} / E_{H}^{*}$ for different values of $\gamma$ all agree extremely well with the earlier variational results). Faulkner noted that $G=$ $\left(E_{p_{ \pm}}-E_{2 p 0}\right) /\left(E_{3 p_{ \pm}}-E_{2 p_{ \pm}}\right)$depends only on $\gamma$. Taking the value of the ratio to be $G=1.543 \pm 0.001$ appropriate for $\mathrm{Si}: \mathrm{P}$ (Fig. 3) gives the value of $\gamma$ shown in Table I. With this value the theoretical splitting $E_{3 p_{ \pm}}-E_{2 p_{ \pm}}=0.08243 E_{H}^{*}$ (and this is very insensitive to $\gamma$; it changes by only $0.1 \%$ over the range $\gamma=0.18-0.22$ ). Taking the experimental $E_{3 p_{ \pm}}-E_{2 p_{ \pm}}=$ $3.283 \pm 0.002 \mathrm{meV}$ appropriate for $\mathrm{Si}: \mathrm{P}$ (Fig. 3) results in the value of $E_{H}^{*}$ shown in Table I. Faulkner's values of $\gamma=0.2079$ and $E_{H}^{*}=39.89 \mathrm{meV}$ (from $G=1.555$ and $E_{3 p \pm}-E_{2 p \pm}=$ $3.28 \mathrm{meV}$ ) are very slightly different simply through use of better samples with sharper lines here. There is a very small variation among species $(\sim 1 \%)$ in both experimental parameters on Fig. 3 though the values for Si:P from the different experiments (this paper and Ref. [46]) are remarkably consistent (differing by $0.05 \%$ ). It is difficult to see a pattern in the values, and although the error bars are in some cases quite large compared with the variation, the case of $\mathrm{Si}: \mathrm{Li}$ is notably different from Si:P within their respective error bars (other species having larger error due to the broader, weaker lines), which is probably due to the (very small but detectable) effects of the CCC on the excited states concerned.

Faulkner took $m_{t}^{*}=0.1905$ (the band-edge value from Hensel's earlier cyclotron resonance of free electrons [20]) and used his value of $E_{H}^{*}$ to extract $\epsilon_{r}$. Using our result for $m_{t}^{*}$ from the LZE from the same experiment (see above) seems
TABLE III. Single-valley state dimensions from EMT produced with the Lanczos method in zero field. The quantities listed are the binding energy, $t=\sqrt{\left\langle x^{2}\right\rangle}=\sqrt{\left\langle y^{2}\right\rangle}, l=\sqrt{\left\langle z^{2}\right\rangle}, r=\sqrt{2 t^{2}+l^{2}}$, $\rho_{0}=\sqrt{2} t$, and $\gamma^{\prime}=l^{2} / t^{2}$. The effective mass parameters used were from Table I. The $1 s$ state mentioned is the single-valley EMT state (ignoring the $\mathrm{CCC}$ ).

\begin{tabular}{lrrrrrc}
\hline \hline & $-E(\mathrm{meV})$ & $t(\mathrm{~nm})$ & $l(\mathrm{~nm})$ & $r(\mathrm{~nm})$ & $\rho_{0}(\mathrm{~nm})$ & $\gamma^{\prime}\left(=l^{2} / t^{2}\right)$ \\
\hline $1 s$ & 30.539 & 2.41 & 1.33 & 3.66 & 3.40 & 0.31 \\
$2 p_{0}$ & 11.463 & 4.42 & 4.48 & 7.69 & 6.25 & 1.02 \\
$3 p_{0}$ & 5.468 & 8.59 & 11.59 & 16.79 & 12.15 & 1.82 \\
$4 p_{0}$ & 3.297 & 12.36 & 21.42 & 27.65 & 17.49 & 3.00 \\
$4 f_{0}$ & 2.330 & 22.45 & 15.09 & 35.15 & 31.75 & 0.45 \\
$5 p_{0}$ & 2.225 & 16.97 & 32.96 & 40.78 & 24.01 & 3.77 \\
$6 p_{0}$ & 1.623 & 21.55 & 48.03 & 56.89 & 30.48 & 4.97 \\
$5 f_{0}$ & 1.504 & 35.33 & 27.99 & 57.27 & 49.96 & 0.63 \\
$2 p_{ \pm}$ & 6.396 & 9.18 & 3.85 & 13.54 & 12.98 & 0.18 \\
$3 p_{ \pm}$ & 3.113 & 17.83 & 13.04 & 28.39 & 25.21 & 0.54 \\
$4 p_{ \pm}$ & 2.181 & 23.37 & 16.69 & 37.02 & 33.05 & 0.51 \\
$4 f_{ \pm}$ & 1.887 & 28.86 & 23.56 & 47.13 & 40.81 & 0.67 \\
$5 p_{ \pm}$ & 1.445 & 33.28 & 36.69 & 59.67 & 47.06 & 1.22 \\
$5 f_{ \pm}$ & 1.255 & 45.10 & 33.51 & 72.04 & 63.77 & 0.55 \\
$6 p_{ \pm}$ & 1.067 & 41.72 & 53.82 & 79.86 & 59.01 & 1.66 \\
\hline \hline
\end{tabular}

preferable for self-consistency, and results in the values of $\epsilon_{r}, a_{B}^{*}$, etc., shown in Table I.

Having determined the mass parameters, we calculated the eigenvalues of $\hat{H}_{0 z}+\hat{H}_{1}+\hat{H}_{2}$ as a function of magnetic field along the valley axis with the Lanczos procedure. We extracted the transverse radius from the excited-state wave functions: The open symbols in Fig. 2(b) show $\rho^{2}=\left\langle x^{2}+y^{2}\right\rangle$ at a range of fields, and the zero-field values are given in Table III. Very small differences in the EMT binding energies between Table III and elsewhere [14] are mainly due to slight differences in parameters in Table II. The ordering of the state labels in Table III is the traditional one [14]. There are two ways to find the theoretical effective transverse radius $\tilde{\rho}$ at zero field. First we calculated the effective radius $\tilde{\rho}^{2}(B)$ from the double derivative of the EMT results for $E(B)$ using Eq. (3) [Fig. 2(b), solid lines], and the zero-field value agrees very well with the zero-field value of the theoretical $\rho$ [Fig. 2(b), open symbols] as expected, which confirms the validity of Eqs. (2) and (3). Then, to assess the procedure that was used to find the experimental $\rho_{0}$ we performed a fit of Eq. (4) to the theoretical $E(B)$. In this case for the $2 p_{ \pm}$and $3 p_{ \pm}$we obtained $\rho_{0}=4.08$ and 7.86 atomic units, i.e., 12.8 and $24.7 \mathrm{~nm}$, respectively. These values agree very well with the experimental values given in Sec. IID above from the fits of Eq. (4) to the experimental data, but are about $1 \%$ and $2 \%$ less, respectively, than the exact $\rho_{0}$ obtained from the theoretical wave functions (Table III). This discrepancy is too small to be visible on Fig. 2(b). It arises just because of the fact that our fitting process took the QZE radius constriction with field, $\tilde{\rho}(B)$, to be a Lorentzian function, which is an imperfect approximation. There may also be an additional systematic error in the experimentally extracted values due to the fact we neglected the contribution of the ground state (which would raise the experimental results by about $1 \%$ and $0.2 \%$, respectively). 


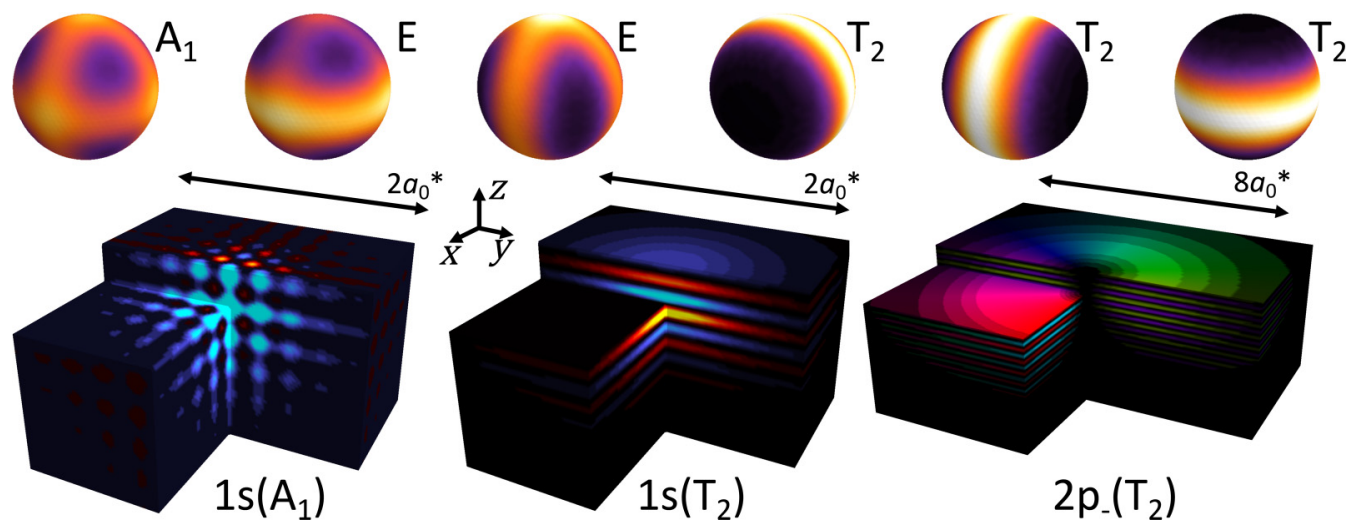

FIG. 4. The wave functions. The top row shows the six ground-state envelope functions: The color scale shows the probability density increasing from black (zero density) to white, on a spherical surface around the donor averaged over a valley interference oscillation period. The bottom row shows some example wave functions including the valley interference term. The brightness shows the probability density on some illustrative surfaces around the donor, and the color scale shows the wave-function phase. The donor is at the origin at the central vertex of the image and the length scale is shown in units of $a_{0}^{*}$. The $1 s\left(T_{2}\right)$ state shown is the $z$-valley component (last one on the top row). All illustrations take the lattice periodic part of the wavefunction $u(\mathbf{r})=1$ simplicity.

\section{DISCUSSION}

We return to the motivation for this paper, which was to examine the possibility for engineering overlap between neighboring impurities for the purposes of quantum information applications. So far in this paper we considered only the slowly varying envelope function, and the need for obtaining high precision values for its radius. It is also important to remember that the wave function is modulated by quickly varying terms, and that these terms interfere for multi-valley wave functions [22,23]. Since valley interference is more commonly discussed with respect to the different components of the $1 s$ ground state, we illustrate the point with those states first. The single valley $1 s$ states are mixed by the CCC and their degeneracy is lifted, and (apart from $\mathrm{Si}: \mathrm{Li}$ ) the ground state has $A_{1}$ symmetry, meaning that the final wave function has the form $\Psi_{1 s(A 1)}(\mathbf{r})=\frac{1}{\sqrt{ } 6} \sum_{\mu} \psi_{1 s, \mu}(\mathbf{r})=\frac{1}{\sqrt{ } 6} \sum_{\nu} \cos \left(k_{0} x_{v}\right) f_{1 s, v}(\mathbf{r})$, where $v$ runs over $x, y, z$, i.e., there is a fast-oscillating, cosinusoidal term in each of the three dimensions. This state is, therefore, quickly oscillating in all three dimensions, as shown in Fig. 4 (bottom left). Note that for the ease of illustration we set the lattice-periodic functions $u(\mathbf{r})=1$ since they do not affect the general conclusions. Wave-function plots for $1 s\left(A_{1}\right)$ with fewer approximations are available elsewhere [22,23]. The conduction band minima are $85 \%$ of the way from the $\Gamma$ to the $X$ point, i.e., $k_{0}=0.85 \pi / a$ and so the $\cos \left(k_{0} x_{v}\right)$ oscillation does not repeat with the lattice spacing. This means that there is a large change in wave-function amplitude from atomic site to atomic site, which makes control over the overlaps difficult because they are extremely sensitive to position errors. We point out now that a significant advantage can be obtained by using excited states to produce the coupling, e.g., using the Stoneham-Fisher-Greenland scheme [6]. The advantage arises from the different valley interference. The high-energy components of the ground state have $E$ or $T_{2}$ symmetry, and form a doublet and a triplet, respectively. The three $T_{2}$ states are quickly varying due to the valley interference in only one direction each: for example, one is $\Psi_{1 s\left(T_{2} z\right)}(\mathbf{r})=$ $\sqrt{2} \sin \left(k_{0} z\right) f_{1 s, z}(\mathbf{r})$, shown in Fig. 4 (bottom middle). These $T_{2}$ states are optically accessible from $1 s\left(A_{1}\right)$ for light polarized along $z$, although with much smaller oscillator strength than to the excited states with odd-parity envelopes such as the $2 p_{-}$state. These $T_{2}$ states would be very forgiving of position errors in two of the three dimensions ( $x$ and $y$ in the case of the $\Psi_{1 s\left(T_{2} z\right)}$ state illustrated). The $E$ states oscillate with $k_{0}$ in two dimensions $(x, y)$ and are therefore forgiving of positioning errors in the other dimension $(z)$. Returning to the odd-parity excited states of our experiment above, the same advantage is obtained for the $2 p_{-}$state in the $z$ valley, $\Psi_{2 p_{-}\left(T_{2} z\right)}(\mathbf{r})=\sqrt{2} \cos \left(k_{0} z\right) f_{2 p_{-}, z}(\mathbf{r})$, which is also varying in only the $z$ direction (Fig. 4, bottom right), and likewise the $2 p_{+}$state. Note that the envelope $f_{2 p_{-}, z}(r)$ is the same as the single-valley EMT used earlier, since the CCC has no effect on excited states. It appears there is a strong motivation for utilizing the coupling between impurities mediated by $\mathrm{THz}$ pulses polarized in the plane, such as in schemes where the atoms in the ground $1 s\left(A_{1}\right)$ state are well isolated from each other, but during their excursion into the excited state they interact [6]. There may even be good reasons to investigate further donor species for which $1 s\left(E, T_{2}\right)$ is the ground state, such as Si:Li.

\section{CONCLUSION}

In conclusion, we have measured the silicon donor excitedstate radii experimentally. We found an analytic form for the field dependence of the radius that fits the data very well, and agrees also with the results of a Lanczos solution to effective mass theory validating the EMT scaling rules. We showed that nonparabolicity effects become detectable above about $10 \mathrm{~T}$, and indeed that there is a detectable $(0.7 \%)$ difference in the zero-field effective masses for the $2 p_{ \pm}$and $3 p_{ \pm}$states due to the higher-frequency Fourier components in more tightly bound states. We provide high precision effective mass parameters for low (and zero) field. The excited-state radii do not vary by more than $2 \%$ among a wide variety of species including double 
donors, and they provide a major advantages for donor-donor coupling due to the more favorable valley interference effects.

The supporting data for this article are openly available from the Zenodo data archive [47].

\section{ACKNOWLEDGMENTS}

We acknowledge financial support from the UK Engineering and Physical Sciences Research Council (ADDRFSS,
Grant Reference No. EP/H001905/1). The work at Nijmegen was performed as part of the research programme of the Stichting voor Fundamenteel Onderzoek der Materie (FOM), which is financially supported by the Nederlandse Organisatie voor Wetenschappelijk Onderzoek (NWO). Work on sample preparation is partially supported by the Russian Academy of Science according to Program No.8 "Physics of condensed matter and new materials". We would also like to thank Dr. Ellis Bowyer for help with some of the experiments.
[1] J. P. Dehollain, S. Simmons, J. T. Muhonen, R. Kalra, A. Laucht, F. Hudson, K. M. Itoh, D. N. Jamieson, J. C. McCallum, A. S. Dzurak, and A. Morello, Bell's inequality violation with spins in silicon, Nat. Nano. 11, 242 (2016).

[2] J. Salfi, J. A. Mol, R. Rahman, G. Klimeck, M. Y. Simmons, L. C. L. Hollenberg, and S. Rogge, Quantum simulation of the Hubbard model with dopant atoms in silicon, Nat. Commun. 7, 11342 (2016).

[3] N. H. Le, A. J. Fisher, and E. Ginossar, Extended Hubbard model for mesoscopic transport in donor arrays in silicon, Phys. Rev. B 96, 245406 (2017).

[4] W. Wu, P. T. Greenland, A. J. Fisher, N. H. Le, S. Chick, and B. N. Murdin, Excited states of defect linear arrays in silicon: A first-principles study based on hydrogen cluster analogs, Phys. Rev. B 97, 035205 (2018).

[5] T. Kobayashi, J. van der Heijden, M. G. House, S. J. Hile, P. Asshoff, M. F. Gonzalez-Zalba, M. Vinet, M. Y. Simmons, and S. Rogge, Resonant tunneling spectroscopy of valley eigenstates on a donor-quantum dot coupled system, Appl. Phys. Lett. 108, 152102 (2016).

[6] A. M. Stoneham, A. J. Fisher, and P. T. Greenland, Optically driven silicon-based quantum gates with potential for hightemperature operation, J. Phys. Condens. Matter 15, L447 (2003).

[7] C. D. Hill, E. Peretz, S. J. Hile, M. G. House, M. Fuechsle, S. Rogge, M. Y. Simmons, and L. C. L. Hollenberg, A surface code quantum computer in silicon, Sci. Adv. 1, e1500707 (2015).

[8] G. Pica, B. W. Lovett, R. N. Bhatt, T. Schenkel, and S. A. Lyon, Surface code architecture for donors and dots in silicon with imprecise and nonuniform qubit couplings, Phys. Rev. B 93, 035306 (2016).

[9] G. Tosi, F. A. Mohiyaddin, V. Schmitt, S. Tenberg, R. Rahman, G. Klimeck, and A. Morello, Silicon quantum processor with robust long-distance qubit couplings, Nat. Commun. 8, 450 (2017).

[10] S. R. Schofield, N. J. Curson, M. Y. Simmons, F. J. Rueß, T. Hallam, L. Oberbeck, and R. G. Clark, Atomically Precise Placement of Single Dopants in Si, Phys. Rev. Lett. 91, 136104 (2003).

[11] S. R. Schofield, P. Studer, C. F. Hirjibehedin, N. J. Curson, G. Aeppli, and D. R. Bowler, Quantum engineering at the silicon surface using dangling bonds, Nat. Commun. 4, 1649 (2013).

[12] K. Saeedi, M. Szech, P. Dluhy, J. Z. Salvail, K. J. Morse, H. Riemann, N. V. Abrosimov, N. Nötzel, K. L. Litvinenko, B. N. Murdin, and M. L. W. Thewalt, Optical pumping and readout of bismuth hyperfine states in silicon for atomic clock applications, Sci. Rep. 5, 10493 (2015).
[13] K. J. Morse, R. J. S. Abraham, A. DeAbreu, C. Bowness, T. S. Richards, H. Riemann, N. V. Abrosimov, P. Becker, H.-J. Pohl, M. L. W. Thewalt, and S. Simmons, A photonic platform for donor spin qubits in silicon, Sci. Adv. 3, e1700930 (2017).

[14] B. Pajot, Donor and donor-like EM spectra, in Optical Absorption of Impurities and Defects in Semiconducting Crystals: Hydrogen-Like Centres (Springer, Berlin, 2009).

[15] S. Debnath, N. M. Linke, C. Figgatt, K. A. Landsman, K. Wright, and C. Monroe, Demonstration of a small programmable quantum computer with atomic qubits, Nature (London) 536, 63 (2016).

[16] G. A. Thomas, M. Capizzi, F. DeRosa, R. N. Bhatt, and T. M. Rice, Optical study of interacting donors in semiconductors Phys. Rev. B 23, 5472 (1981).

[17] R. H. Garstang, Atoms in high magnetic-fields, Rep. Prog. Phys. 40, 105 (1977).

[18] T. Pohl, H. R. Sadeghpour, and P. Schmelcher, Cold and ultracold Rydberg atoms in strong magnetic fields, Phys. Rep. 484, 181 (2009).

[19] W. Kohn and J. M. Luttinger, Theory of donor states in silicon, Phys. Rev. 98, 915 (1955).

[20] J. C. Hensel, H. Hasegawa, and M. Nakayama, Cyclotron resonance in uniaxially stressed silicon. II. Nature of the covalent bond, Phys. Rev. 138, A225 (1965).

[21] R. A. Faulkner, Higher donor excited states for prolate-spheroid conduction bands: A reevaluation of silicon and germanium, Phys. Rev. 184, 713 (1969).

[22] C. J. Wellard and L. C. L. Hollenberg, Donor electron wave functions for phosphorus in silicon: Beyond effective-mass theory, Phys. Rev. B 72, 085202 (2005).

[23] J. K. Gamble, N. T. Jacobson, E. Nielsen, A. D. Baczewski, J. E. Moussa, I. Montano and R. P. Muller, Multivalley effective mass theory simulation of donors in silicon, Phys. Rev. B 91, 235318 (2015).

[24] G. Feher, Electron spin resonance experiments on donors in silicon. I. Electronic structure of donors by the electron nuclear double resonance technique, Phys. Rev. 114, 1219 (1959).

[25] E. B. Hale and R. L. Mieher, Shallow donor electrons in silicon. I. Hyperfine interactions from ENDOR measurements, Phys. Rev. 184, 739 (1969).

[26] G. Pica, G. Wolfowicz, M. Urdampilleta, M. L. W. Thewalt, H. Riemann, N. V. Abrosimov, P. Becker, H.-J. Pohl, J. J. L. Morton, R. N. Bhatt, S. A. Lyon, and B. W. Lovett, Hyperfine Stark effect of shallow donors in silicon, Phys. Rev. B 90, 195204 (2014).

[27] K. Sinthiptharakoon, S. R. Schofield, P. Studer, V. Brázdová, C. F. Hirjibehedin, D. R. Bowler and N. J. Curson, Investigating individual arsenic dopant atoms in silicon using low-temperature 
scanning tunnelling microscopy, J. Phys.: Condens. Matter 26, 012001 (2014).

[28] J. Salfi, J. A. Mol, R. Rahman, G. Klimeck, M. Y. Simmons, L. C. L. Hollenberg, and S. Rogge, Spatially resolving valley quantum interference of a donor in silicon, Nat. Mater. 13, 605 (2014).

[29] M. Usman, J. Bocquel, J. Salfi, B. Voisin, A. Tankasala, R. Rahman, M. Y. Simmons, S. Rogge, and L. C. L. Hollenberg, Spatial metrology of dopants in silicon with exact lattice site precision, Nat. Nanotechnol. 11, 763 (2016).

[30] V. Brazdova, D. R. Bowler, K. Sinthiptharakoon, P. Studer, A. Rahnejat, N. J. Curson, S. R. Schofield, and A. J. Fisher, Exact location of dopants below the $\mathrm{Si}(001)$ : $\mathrm{H}$ surface from scanning tunnelling microscopy and density functional theory, Phys. Rev. B 95, 075408 (2017).

[31] A. Patanè, N. Mori, O. Makarovsky, L. Eaves, M. L. Zambrano, J. C. Arce, L. Dickinson, and D. K. Maude, Manipulating and Imaging the Shape of an Electronic Wave Function by Magnetotunneling Spectroscopy, Phys. Rev. Lett. 105, 236804 (2010).

[32] W. Lei, C. Notthoff, J. Peng, D. Reuter, A. Wieck, G. Bester, and A. Lorke, Artificial Atoms in Magnetic Fields: Wave-Function Shaping and Phase-Sensitive Tunneling, Phys. Rev. Lett. 105, 176804 (2010).

[33] K. L. Litvinenko, M. Pang, J. Li, E. Bowyer, H. Engelkamp, V. B. Shuman, L. M. Portsel, A. N. Lodygin, Yu. A. Astrov, S. G. Pavlov, H.-W. Hübers, C. R. Pidgeon, and B. N. Murdin, High-field impurity magneto-optics of Si:Se, Phys. Rev. B 90, 115204 (2014).

[34] K. L. Litvinenko, J. Li, N. Stavrias, A. J. Meaney, P. C. M. Christianen, H. Engelkamp, K. P. Homewood, C. R. Pidgeon, and B. N. Murdin, The quadratic Zeeman effect used for state-radius determination in neutral donors and donor bound excitons in Si:P, Semicond. Sci. Technol. 31, 045007 (2016).

[35] B. N. Murdin, J. Li, M. L. Y. Pang, E. T. Bowyer, K. L. Litvinenko, S. K. Clowes, H. Engelkamp, C. R. Pidgeon, I. Galbraith, N. V. Abrosimov, H. Riemann, S. G. Pavlov, H.-W. Hübers, and P. G. Murdin, Si:P as a laboratory analogue for hydrogen on high magnetic field white dwarf stars, Nat. Commun. 4, 1469 (2013).

[36] R. A. Lewis, A. Bruno-Alfonso, G. V. B. de Souza, R. E. M. Vickers, J. A. Colla, and E. Constable, Spherical, cylindrical and tetrahedral symmetries; hydrogenic states at high magnetic field in Si:P, Sci. Rep. 3, 3488 (2013).

[37] Yu. A. Astrov, L. M. Portsel, A. N. Lodygin, and V. B. Shuman, Gas-phase doping of silicon with sulfur, Semicond. Sci. Technol. 26, 055021 (2011).

[38] Yu. A. Astrov, L. M. Portsel, A. N. Lodygin, V. B. Shuman and N. V. Abrosimov, Planar sulfur-doped silicon detectors for high-speed infrared thermography, Infrared Phys. Technol. 52, 25 (2009).

[39] Yu. A. Astrov, V. B. Shuman, A. N. Lodygin, L. M. Portsel, and A. N. Makhova, Development of photodetectors for image converters: Doping of silicon with selenium from the gas phase, Semiconductors 42, 448 (2008).

[40] Yu. A. Astrov, V. B. Shuman, L. M. Portsel, A. N. Lodygin, S. G. Pavlov, N. V. Abrosimov, V. N. Shastin, and H.-W. Hübers, Diffusion doping of silicon with magnesium, Phys. Status Solidi A 214, 1700192 (2017).

[41] T. G. Castner, Jr., Tetrahedral potentials and lack-of-inversion symmetry for donors in silicon, Phys. Rev. B 79, 195207 (2009).

[42] B. Pajot, F. Merlet, and G. Taravella, Quadratic Zeeman Effect of donor lines in silicon. II. Comparison with experiment, Can. J. Phys. 50, 2186 (1972).

[43] M. Diarra, Y.-M. Niquet, C. Delerue, and G. Allan, Ionization energy of donor and acceptor impurities in semiconductor nanowires: Importance of dielectric confinement, Phys. Rev. B 75, 045301 (2007).

[44] Y. M. Niquet, D. Rideau, C. Tavernier, H. Jaouen, and X. Blase, On site matrix elements of the tight-binding Hamiltonian of a strained crystal: Application to silicon, germanium, and their alloys, Phys. Rev. B 79, 245201 (2009).

[45] J. Mansir, P. Conti, Z. Zeng, J. J. Pla, P. Bertet, M. W. Swift, C. G. Van de Walle, M. L. W. Thewalt, B. Sklenard, Y. M. Niquet, and J. J. L. Morton, Linear Hyperfine Tuning of Donor Spins in Silicon Using Hydrostatic Strain, Phys. Rev. Lett. 120, 167701 (2018).

[46] M. Steger, A. Yang, D. Karaiskaj, M. L. W. Thewalt, E. E. Haller, J. W. Ager, III, M. Cardona, H. Riemann, N. V. Abrosimov, A. V. Gusev, A. D. Bulanov, A. K. Kaliteevskii, O. N. Godisov, P. Becker, and H.-J. Pohl, Shallow impurity absorption spectroscopy in isotopically enriched silicon, Phys. Rev. B 79, 205210 (2009).

[47] http://doi.org/10.5281/zenodo.1338126. 